\title{
REMARKS ON SOME ASPECTS OF SPECTRAL CLASSIFICATION
}

\author{
W. W. MORGAN \\ Yerkes Observatory, University of Chicago, U.S.A.
}

Abstract. The present state of MK spectral classification is described briefly.

\section{Introduction}

It is now over 30 years since the publication of the MKK Atlas of Stellar Spectra; and the time has come for a general re-examination of the mission of spectral classification. The principles outlined in the Introduction to the Yerkes Atlas of 1943 still seem valid; but the classification problem has become more complex in the intervening years through new astrophysical discoveries - and through the rapid growth of spectral classification by narrow-band photoelectric photometry (see, for example, B. Strömgren 1966, Ann. Rev. Astron. Astrophys. 4, 433).

Such a re-examination is now in progress in two separate works: (1) the preparation of a revised atlas of spectral classification for the stars of later type, by Keenan; and (2) a new atlas of stellar spectra for stars earlier than the Sun, by Abt and Morgan. In the case of the latter work a major increase in the precision with which the MK system is defined is being achieved; this increase in systemic accuracy has already led to the discovery of several new categories of 'peculiar' stellar spectra.

An interesting example of a 'peculiar' category has been noted recently by Abt and Morgan for a group of metallic-line stars having rather strong $\mathrm{K}$-line intensities. Members of this group, for which the Am star HD 103877 can be considered a prototype, have metallic-line types around F3-F5, and luminosity classes IV or V, in the neighbourhood of $\lambda$ 4300. On the other hand, in the spectral range $\lambda \lambda 3850-4100$ the luminosity classes range from Ib to III. In the case of the prototype, the spectrum in the latter region resembles closely that of $\alpha$ Persei (F5 Ib) on spectrograms of scale near $125 \AA \mathrm{mm}^{-1}$, except for a weaker $\mathrm{K}$ line. Thus, we can say that the spectrum in the violet region noted does not correspond to that of a main-sequence star of any spectral type (Abt and Morgan: manuscript in preparation).

\section{The Migration of Types over the Spectral Type - Luminosity Class Diagram}

Some years ago, it was the custom to define spectral class $O$ by stars whose spectra contain lines of He II. As the quality of spectrograms improved, more and more B0 stars were found to contain He II lines - and thus were moved into class O. Occurrences such as this emphasize the need for defining spectral types and luminosity classes in terms of standard stars. This is one of the principal features of the MKK Atlas of 1943. 
The need for some such procedure is not always realized at the present time; 'revised' MK types are still being published by some investigators in which the fundamental standards which define the MK system are given new values. Such procedures result in a floating classfication system, where absolute determinations of types result and the differential advantage of MK classification is lost. But this does not at all imply that the MKK or MK standards are sacrosanct; some of them have been changed in the past; and more are being changed in the present, in connection with the preparation of the new atlases. But it is important to specify a new set of standard stars when the MK standards are altered. If this is not done, the noise level is raised over the entire two-dimensional diagram.

\section{The Mission of Spectral Classification in the Near Future}

Among other ongoing projects which are concerned with fundamental spectral classification, we note one of prime importance: the monumental Michigan Catalogue of TwoDimensional Spectral Types for the Henry Draper Stars, where the principal classification is being carried out by Dr Nancy Houk. The first volume, which includes types from the South Pole to $-53^{\circ}$ declination, will emerge from press in the very near future. This project sets a new epoch in spectral classification and establishes its principal classifier as in the strain of such figures of the past as Miss Annie Cannon - and the greatest of all spectral classifiers, Miss Antonia C. Maury. The completion of the Michigan Catalogue is the most pressing of all the classification projects. It will furnish a wealth of new material for investigations of Galactic structure - and for astrophysical research. The greatest problem remaining for it is how the plates for the Northern fields are to be obtained.

The most important task for the revised MK atlases of Keenan and of Abt and Morgan is to improve the systemic precision to the greatest degree practicable. This will result in more accurate spectroscopic luminosities and parallaxes on one hand, and the identification and delineation of new categories of objects of astrophysical interest. Each atlas should be accompanied by an extensive catalogue of stars classified according to the new precepts - and, in effect, defining the revised system illustrated. 